Research

\title{
Engineering bacteria to solve the Burnt Pancake Problem
} Karmella A Haynes*1, Marian L Broderick ${ }^{4}$, Adam D Brown ${ }^{3}$, Trevor L Butner ${ }^{3}$, James O Dickson ${ }^{2}$, W Lance Harden², Lane H Heard ${ }^{3,6}$, Eric L Jessen ${ }^{3}$, Kelly J Malloy ${ }^{3}$, Brad J Ogden ${ }^{2}$, Sabriya Rosemond ${ }^{1,5}$, Samantha Simpson ${ }^{1}$, Erin Zwack ${ }^{1}$, A Malcolm Campbell ${ }^{1}$, Todd T Eckdahl ${ }^{3}$, Laurie J Heyer ${ }^{2}$ and Jeffrey L Poet ${ }^{4}$

Address: ${ }^{1}$ Davidson College, Department of Biology, Davidson, NC 28036, USA, ${ }^{2}$ Davidson College, Department of Mathematics, Davidson, NC 28036, USA, ${ }^{3}$ Missouri Western State University, Department of Biology, St. Joseph, MO 64507, USA, ${ }^{4}$ Missouri Western State University, Department of Computer Science, Math and Physics, St. Joseph, MO 64507, USA, ${ }^{5}$ Hampton University, Biology Department, Hampton, VA 23668, USA and ${ }^{6}$ Central High School, St. Joseph, MO 64506, USA

Email: Karmella A Haynes* - karmella@gmail.com; Marian L Broderick - mlb8172@missouriwestern.edu; Adam D Brown - adb1207@missouriwestern.edu; Trevor L Butner - tlb3238@missouriwestern.edu; James O Dickson - jidickson@davidson.edu; W Lance Harden - laharden@davidson.edu; Lane H Heard - axenmoon@hotmail.com; Eric L Jessen - elj0152@missouriwestern.edu; Kelly J Malloy - kjm6938@stowers-institute.org; Brad J Ogden - bjo3000@missouriwestern.edu; Sabriya Rosemond - sabriyarosemond@yahoo.com; Samantha Simpson - sasimpson@davidson.edu; Erin Zwack - erzwack@davidson.edu; A Malcolm Campbell - macampbell@davidson.edu; Todd T Eckdahl - eckdahl@missouriwestern.edu; Laurie J Heyer - laheyer@davidson.edu; Jeffrey L Poet - poet@missouriwestern.edu

* Corresponding author

Published: 20 May 2008

Journal of Biological Engineering 2008, 2:8 doi:10.1186/1754-1611-2-8
Received: 9 December 2007

Accepted: 20 May 2008

This article is available from: http://www.jbioleng.org/content/2/l/8

(C) 2008 Haynes et al; licensee BioMed Central Ltd.

This is an Open Access article distributed under the terms of the Creative Commons Attribution License (http://creativecommons.org/licenses/by/2.0), which permits unrestricted use, distribution, and reproduction in any medium, provided the original work is properly cited.

\begin{abstract}
Background: We investigated the possibility of executing DNA-based computation in living cells by engineering Escherichia coli to address a classic mathematical puzzle called the Burnt Pancake Problem (BPP). The BPP is solved by sorting a stack of distinct objects (pancakes) into proper order and orientation using the minimum number of manipulations. Each manipulation reverses the order and orientation of one or more adjacent objects in the stack. We have designed a system that uses site-specific DNA recombination to mediate inversions of genetic elements that represent pancakes within plasmid DNA.

Results: Inversions (or "flips") of the DNA fragment pancakes are driven by the Salmonella typhimurium Hin/hix DNA recombinase system that we reconstituted as a collection of modular genetic elements for use in $E$. coli. Our system sorts DNA segments by inversions to produce different permutations of a promoter and a tetracycline resistance coding region; $E$. coli cells become antibiotic resistant when the segments are properly sorted. Hin recombinase can mediate all possible inversion operations on adjacent flippable DNA fragments. Mathematical modeling predicts that the system reaches equilibrium after very few flips, where equal numbers of permutations are randomly sorted and unsorted. Semiquantitative PCR analysis of in vivo flipping suggests that inversion products accumulate on a time scale of hours or days rather than minutes.

Conclusion: The Hin/hix system is a proof-of-concept demonstration of in vivo computation with the potential to be scaled up to accommodate larger and more challenging problems. Hin/hix may provide a flexible new tool for manipulating transgenic DNA in vivo.
\end{abstract}




\section{Background}

The tremendous information storage capacity of DNA and the remarkable efficiency of biomolecular self-assembly have inspired researchers to design biological computers. Previous work has created proof-of-concept biological computers based on in vitro self-assembly of DNA [1] and protein-DNA interactions [2-4]. Thus far, biological computing is limited to nonliving devices that have not utilized the parallel processing power afforded by DNA replication and cellular division. In order to demonstrate the feasibility of in vivo computing, we programmed Escherichia coli to address a classic mathematical challenge called the Burnt Pancake Problem (BPP) [5]. The BPP can be visualized as a stack of different sized pancakes, each having one burnt side and one golden side, arranged in an arbitrary order. The stack must be sorted by flipping individual pancakes or subsets of adjacent pancakes until the pancakes are ordered from smallest to largest with each pancake oriented golden side up (see example in Fig. 1). The BPP is also known as sorting by reversals since both the order and orientation of the pancakes are changed when they are flipped. The BPP is a subject of interest in basic mathematical and computational research (e.g., [5]). Of particular interest to biologists is the application of the BPP to comparative genomics. The evolutionary distance between syntenic genomes of two organisms is determined by the minimum number of reversals required to sort regions of genes in one organism to match the order and orientation of orthologous genes in the other organism [6-8]. The total number of possible arrangements of $n$ objects (i.e., pancakes or genes) is $2^{n}(n !)$, an exponential increase in arrangements as the stack of objects (pancakes or genes) becomes larger. Plasmid DNA replication and exponential cell growth in bac-

A
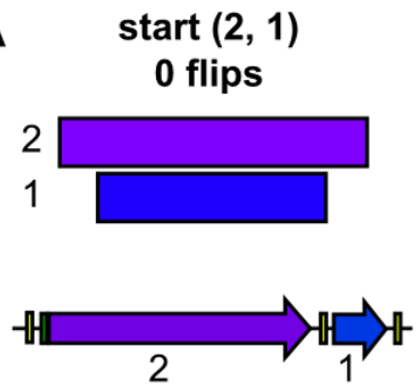

2
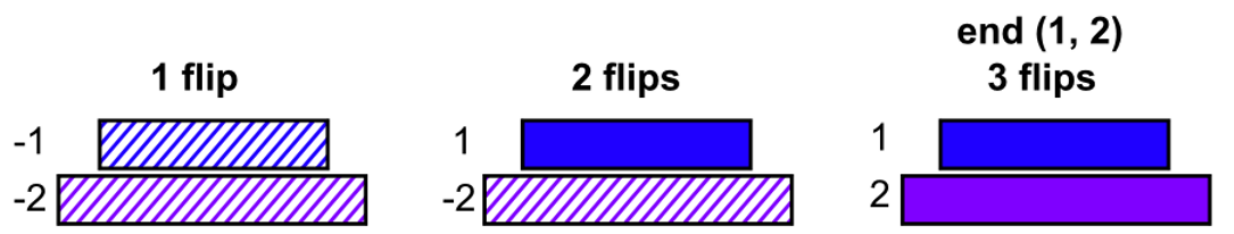

B
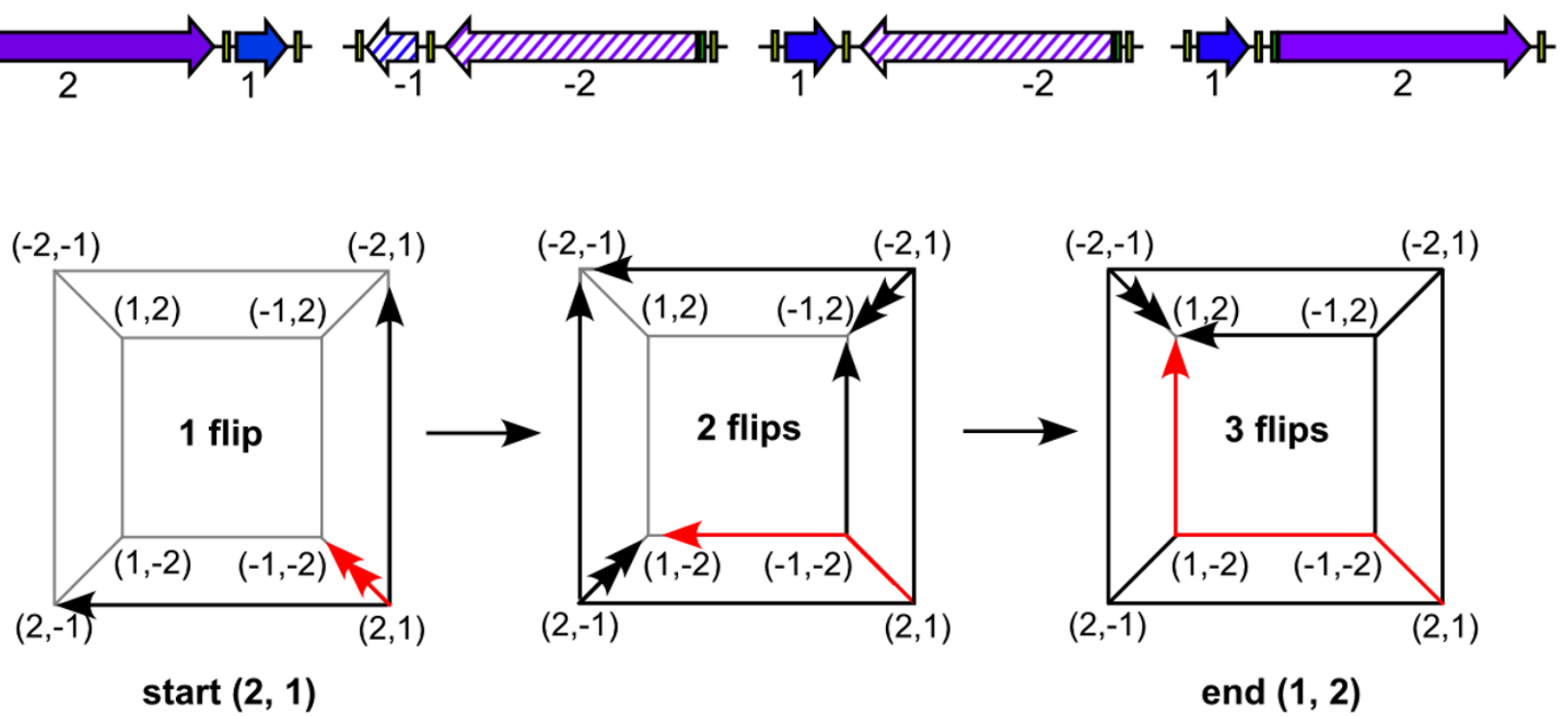

$(-2,1)$
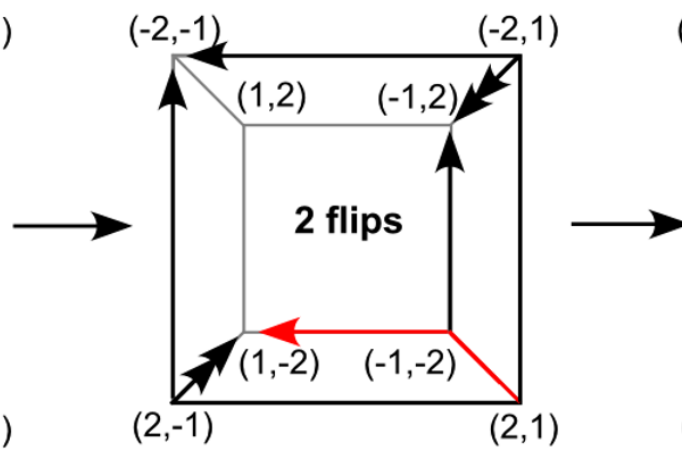

$\left.\right|_{(2,-1)} ^{(-2,-1)}$

$(-2,1)$

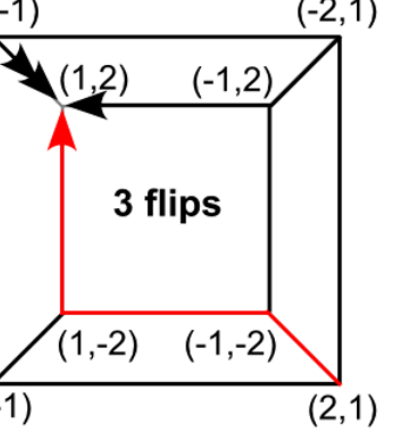

end $(1,2)$

Figure I

The Burnt Pancake Problem can be modeled using genetic elements. (A) Sorting of a scrambled two-pancake stack (rectangles) where the smaller burnt pancake (I, blue) and the larger burnt pancake (2, purple) are in the wrong order (2, I). First, the whole stack is flipped and both pancakes are turned burnt side up (hatched shading). The next two flips turn the small then the large pancake golden side up (solid shading) resulting in a properly sorted stack (I, 2). Analogous DNA segment arrangements are shown below. Sorting of the promoter (I, blue arrow) and coding region (2, purple arrow) into ( 1,2$)$ is required for gene expression. (B) The process of sorting scrambled pancake stack $(2, I)$ into the solution $(I, 2)$ is plotted on a graph. The eight possible arrangements of the pancake stack are shown as signed permutations at the vertices. (2, I) is converted into the three neighboring permutations by a flip of a single pancake (arrow) or both pancakes simultaneously (double headed arrow). Six distinct paths of length 3 can convert $(2, I)$ into $(I, 2)$. The flipping pathway highlighted in red corresponds to the flips shown in part $A$ 
teria are inexpensive, occupy much less space than computer hardware, and maintain parity with the exponential increase in BPP arrangements. Therefore, solving the BPP in living cells offers unique advantages over using computer hardware.

The biological equivalent of a burnt pancake is a functional module of DNA such as a promoter or coding region (Fig. 1a). Similar to burnt pancakes in the BPP, DNA modules have directionality ( $5^{\prime}$ to $\left.3^{\prime}\right)$, require a specific order of the units (e.g., promoter followed by coding region) and can be flipped (cut, inverted, and spliced in vivo by cellular machinery). We designed a modular system in which pancake stacks are assembled from flippable DNA segments. Flipping of the DNA segment "pancakes" is mediated by a Salmonella typhimurium-derived DNA recombination system. In Salmonella, Hin DNA recombinase catalyzes an inversion reaction that regulates the expression of alternative flagellin genes by switching the orientation of a promoter located on a $1 \mathrm{~kb}$ invertible DNA segment $[9,10]$. Two palindromic 26 bp hix sequences flank the invertible DNA segment and serve as the recognition sites for cleavage and strand exchange. A $\sim 70$ bp cis-acting recombinational enhancer $(R E)$ increases efficiency of protein-DNA complex formation [11]. We have reconstituted the genetic elements required for DNA inversion as a collection of modular genetic elements for use in E. coli. Our system is a proof-of-concept genetic computing device that manipulates plasmid DNA processors within living cells.

\section{Results and discussion \\ Design and construction of a Hin/hix-based DNA recombination system}

DNA inversion occurs very rapidly in vitro. Protein-DNA complex assembly, strand cleavage, inversion, and ligation occur in less than 1 minute [11]. Therefore, we engineered Hin/hix inversion to be more tractable to regulation and kinetic studies by decreasing inversion efficiency. Hin was cloned from S. typhimurium by PCR. An ssrA LVA protein degradation tag [12] was added to the Cterminal DNA binding domain to prevent over accumulation of Hin and to achieve tighter control of DNA inversion. In Salmonella, the asymmetrical palindromic sequences hixL and hixR flank the invertible DNA segment and serve as the recognition sites for cleavage and strand exchange. Our system uses hixC, a composite symmetrical hix site that shows higher binding affinity for Hin and a 16-fold slower inversion rate than wild type sites hixL and hixR $[13,14]$.

To build a proof-of-concept model, we designed a twopancake BPP containing the Lac promoter ( $p L a c$ ) and a tetracycline resistance coding region with a ribosomal binding site upstream $(R B S$-tet $A(C))$, each flanked by hix $C$ sites
(Fig. 2). Each configuration of this two-pancake stack is represented by a mathematical signed permutation. For instance, hixC-RBS-tetA(C)-hixC-pLac rev $-h i x C$ is represented as the signed permutation " $(2,-1)$ " where RBStet $A(C)$ is 2 and $p L a c$ is 1 . The positive value (2) represents the forward orientation of RBS-tetA(C) and the negative value $(-1)$ represents the reverse orientation of $p L a c$, denoted $p L a c_{\text {rev }}$ ( $p L a c$ reversed). The eight possible signed permutations can be plotted as vertices of a graph (Fig. 1b). Two signed permutations are connected by an edge if it is possible to convert one permutation to the other with a flip of one or two pancakes. When flipping occurs at random, the starting permutation can be converted into any of its three neighboring permutations. In cells, after a given amount of time (i.e., number of flips), flipping is stopped by manual cell lysis, BPP plasmids are purified and transformed into new cells lacking HinLVA, and solved BPP plasmids are detected by resistance to tetracycline ( $p$ Lac driven RBS-tetA(C) expression) in each colony. The time point at which the BPP is first solved at random reflects the minimal number of flips required to solve the BPP.

\section{HinLVA flips and sorts hixC-flanked DNA segments in vivo} In order to solve the BPP, HinLVA must be able to flip single pancakes of varying sizes, flip adjacent segments independently, and sort segments by flipping multiple pancakes simultaneously. First, we tested HinLVA-mediated inversion on single hixC-flanked DNA segments of different lengths. HinLVA successfully flips the $1212 \mathrm{bp}$ RBS-tet $A(C)$ segment (Fig. 3a). The length of RBS-tetA(C) is comparable to the segment that is inverted by Hin recombinase in Salmonella $[9,10]$. HinLVA can also flip the much shorter 200 bp hixC-flanked promoter (Fig. 3b). Restriction digest fragments indicate approximately equal molar amounts of both conformations (forward and reverse), suggesting that flipping of one DNA pancake has reached equilibrium $\sim 24$ hours after transformation. These data indicate that HinLVA-mediated inversion reconstituted in $E$. coli is not limited by fragment size, at least not within the range of $200-1212 \mathrm{bp}$.

Next, we cotransformed cells with a BPP plasmid containing a hixC-flanked $R B S$-tet $A(C)_{\text {rev }}$ coding region and a hixC-flanked pLac promoter (permutation $(-2,1)$ ) and a HinLVA expression plasmid (Fig. 2). The RE was omitted from the BPP plasmid to slow the rate of inversion. We used multiplex semiquantitative PCR (sqPCR) to monitor flipping of the two adjacent hixC-flanked DNA segments. Each of the four internal rearrangements can be detected by a sqPCR amplicon of a distinct size (Fig. 4a). Eleven hours after transformation, single colonies were picked for whole cell sqPCR. Bands from all four configurations were visible in samples where $(-2,1)$ was cotransformed with HinLVA (Fig. 4b). Flipping occurred in the absence 


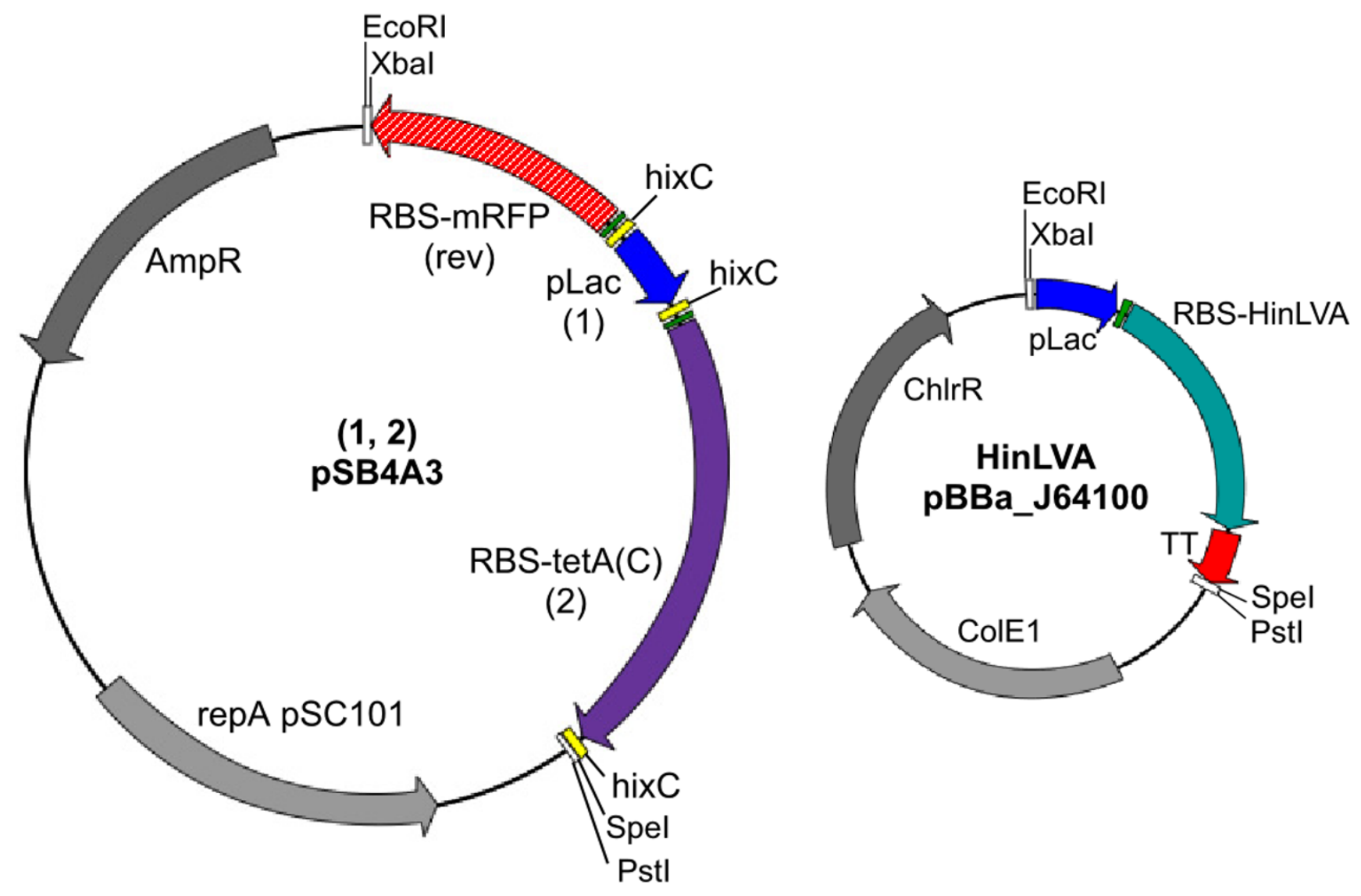

Figure 2

BPP and HinLVA plasmid constructs. A solved BPP plasmid (left) contains a flippable pLac promoter (blue arrow) and RBS-tet $A(C)$ (green rectangle $=R B S$, purple arrow = tet $A(C)$ ). The pLac promoter is pancake I and $R B S$-tet $A(C)$ is pancake 2 . hix $C$ sites (yellow rectangles) flank each flippable element. Inversion of pLac is detected by expression of the reverse (rev) upstream RBS-mRFP reporter. HinLVA is expressed from a second plasmid (right). AmpR = ampicillin resistance marker, ChlrR $=$ chloramphenicol resistance marker, repA PSCIOI and ColEI = origins of replication, $\mathrm{RBS}=$ ribosome binding site, $\mathrm{TT}=\mathrm{double}$ transcription terminator, white boxes = cloning sites.

of the RE, demonstrating that HinLVA and a pair of hixC sites are sufficient for a functional Hin/hix DNA inversion system in E. coli. The starting pancake arrangement $(-2,1)$ is the predominant plasmid in all colonies tested. Plasmids generated from a single flip of either RBS-tetA(C) (pancake 2) or pLac (pancake 1) are the next most frequent, while plasmids generated from two sequential flips of both pancakes 2 and 1 are the least common. We could not detect significant bias for flipping of the larger RBStetA(C) segment or the smaller pLac promoter (Fig. 4c), suggesting that flipping is not influenced by the size of the DNA segment.

The sqPCR results suggest that flipping has not yet reached equilibrium after 11 hours of HinLVA activity in the absence of RE. Plasmid supercoiling might be a limiting factor. Hin-mediated inversion requires a negatively supercoiled plasmid DNA substrate $[15,16]$. The loss of four negative supercoils after each inversion event [17] might require cells to undergo cell division to reset optimal supercoiling before a second inversion event can occur. Based on the 4 hour lag time and 36 minute maximum doubling rate of the cotransformed cells, we estimate that no more than 12 doublings occurred before sqPCR analysis. Twelve cell divisions appear to be insufficient to allow the distribution of rearrangements to reach equilibrium.

Finally, we assessed simultaneous inversion of both DNA pancakes. In order to accomplish this operation, Hin must recognize the outer-most hixC sites and ignore the central hixC site between the segments. Inversion of the entire permutation $(-2,1)$ generates permutation $(-1,2)$ in which the pLac promoter is repositioned to drive $m R F P$ reporter expression (Fig. 5a). Inversion of the promoter alone, producing $(-2,-1)$, is insufficient to induce detect- 

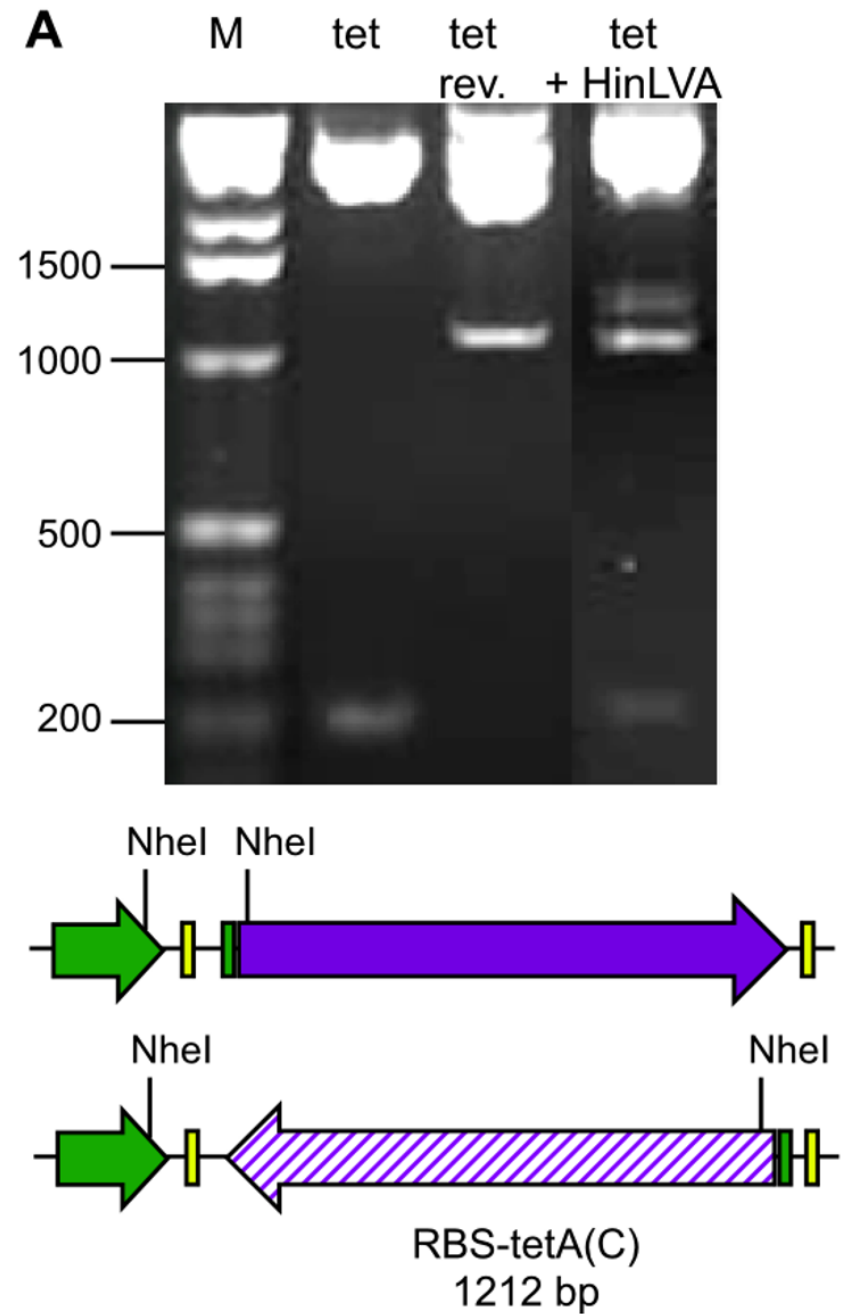
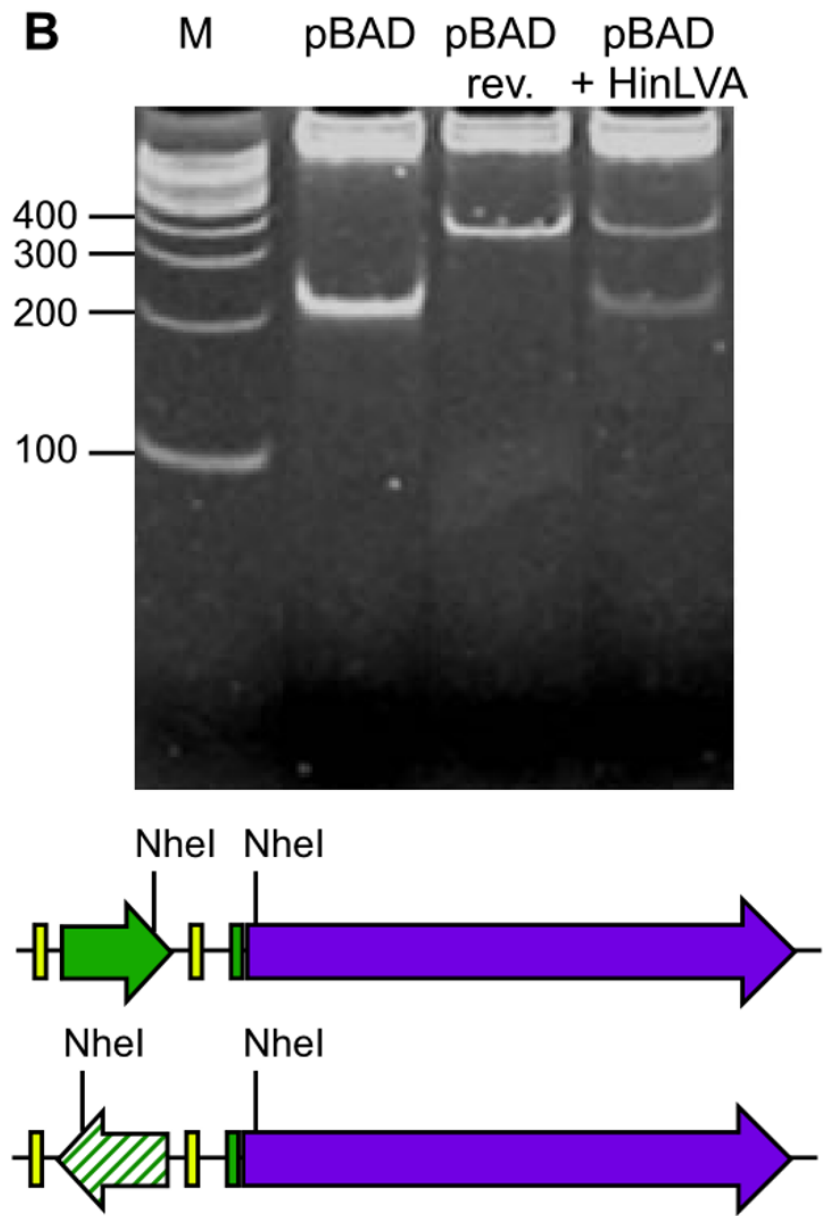

pBAD $200 \mathrm{bp}$

Figure 3

HinLVA mediates inversions of short and long DNA fragments. HinLVA-mediated flipping was assessed by Nhel restriction digests. Gel electrophoresis images are shown in $\mathrm{A}$ and $\mathrm{B}$. (A) $\mathrm{A}$ construct carrying hix $C$-flanked $R B S$-tet $A(C)$ in the forward orientation (solid purple arrow) is characterized by a $\sim 200$ bp Nhel restriction fragment (gel lane 2). The reverse orientation of RBS-tet $A(C)$ (hatched purple arrow) yields a larger 1100 bp band (lane 3). When forward oriented hix C-flanked RBS-tet $A(C)$ is exposed to HinLVA, bands for both forward and reverse orientations of $R B S$-tet $A(C)$ are detected (lane 4). (B) Similarly, forward oriented hixC-flanked PBAD promoter (solid green arrow) is converted to the reverse orientation (hatched green arrow) after exposure to HinLVA (lane 4). These data show that HinLVA-mediated inversion activity is not constrained by the length of hixC-flanked DNA.

able levels of mRFP (Table 1). Colonies containing HinLVA and the $(-2,1)$ BPP plasmid were grown as a liquid culture then the Hin-exposed BPP plasmids were isolated and transformed into bacteria. About one third of the cell colonies appeared red (Fig. 5b) indicating that simultaneous inversion of both DNA segments occurred at a high frequency. Thus, HinLVA is capable of mediating the inversion of at least two adjacent flippable DNA segments.

\section{Modeling and detection of phenotypic output}

We sought to use the power and sensitivity of antibiotic resistance phenotype screening to detect solved BPP plasmids. sqPCR analyzes one colony at a time and requires several plasmids to generate a detectable PCR amplicon, whereas screening can rapidly distinguish a single solved BPP plasmid from millions of unsolved plasmids in a cell culture. Permutations $(1,2)$ and $(-2,-1)$ both encode a functional tetracycline resistance gene that should allow cells to live in the presence of tetracycline. The other six permutations encode a disrupted tetracycline resistance 
A

$(2,-1)$

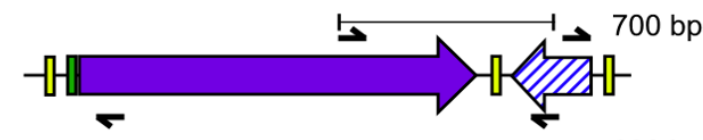

$(2,1)$

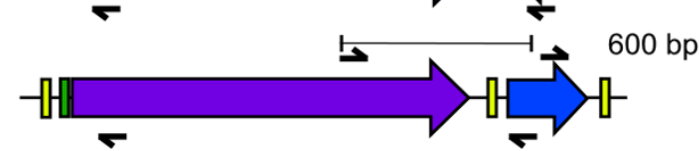

$(-2,-1)$

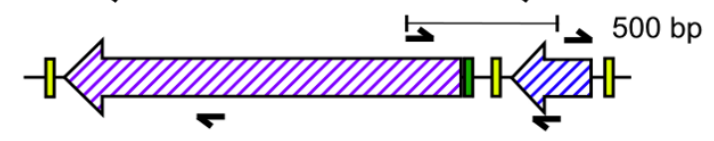

$(-2,1)$

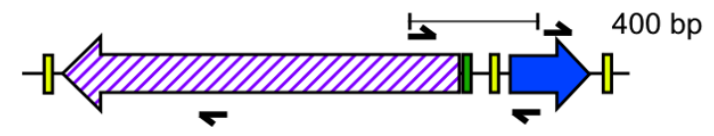

B
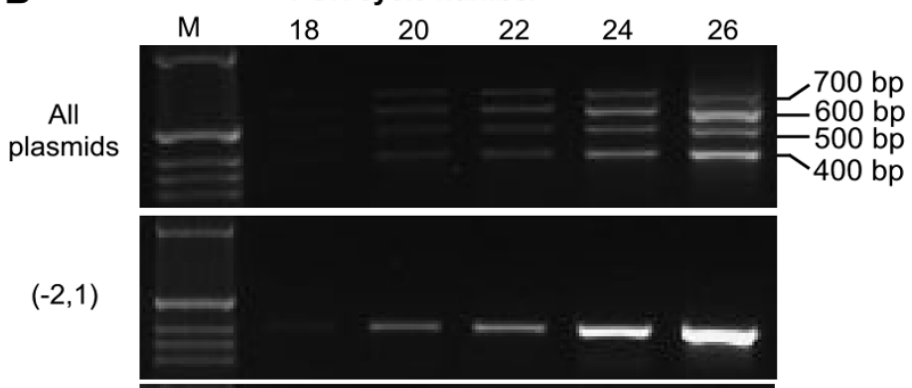

$+\mathrm{Hin}$

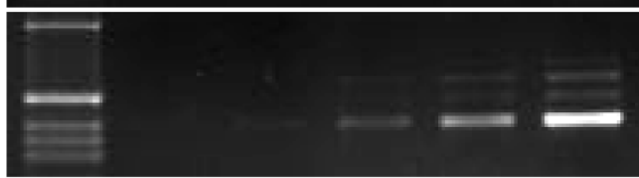

C

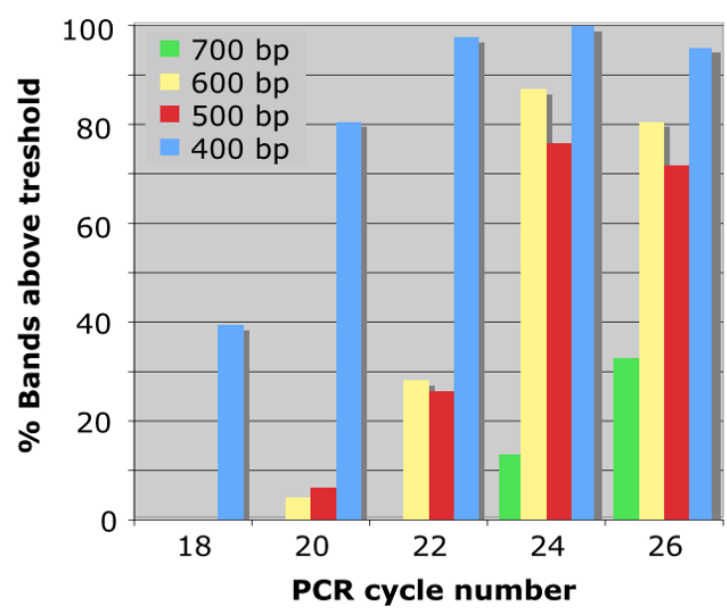

\section{Figure 4}

HinLVA flips adjacent hixC-flanked segments independently. (A) Four pancake stack arrangements can be distinguished by quantitative multiplex PCR. Black arrows indicate primers for multiplex PCR. Bars indicate sizes of PCR amplicons. Whole stack inversions that generate the other four possible arrangements cannot be distinguished in this assay. (B) Quantitative PCR of an equimolar mix of all eight manually assembled BPP plasmids (top row), BPP plasmid (-2, I) alone (middle row), or a single 10-hour colony of cells carrying BPP plasmid $(-2, I)$ in the presence of HinLVA (bottom row). The first lane in each gel contains a DNA molecular weight marker (M). (C) Histogram showing the frequency of detectable bands at I8, 20, 22, 24 and 26 PCR cycles $(n=46$ for each band at each cycle). At $10-I I$ hours following cotransformation of $(-2, I)$ and HinLVA, the starting pancake arrangement predominates (400 bp band). A single flip of either pancake alone (producing a 500 bp or a 600 bp amplicon) is next most frequent occurrence, while two successive flips (700 bp) is least common. 

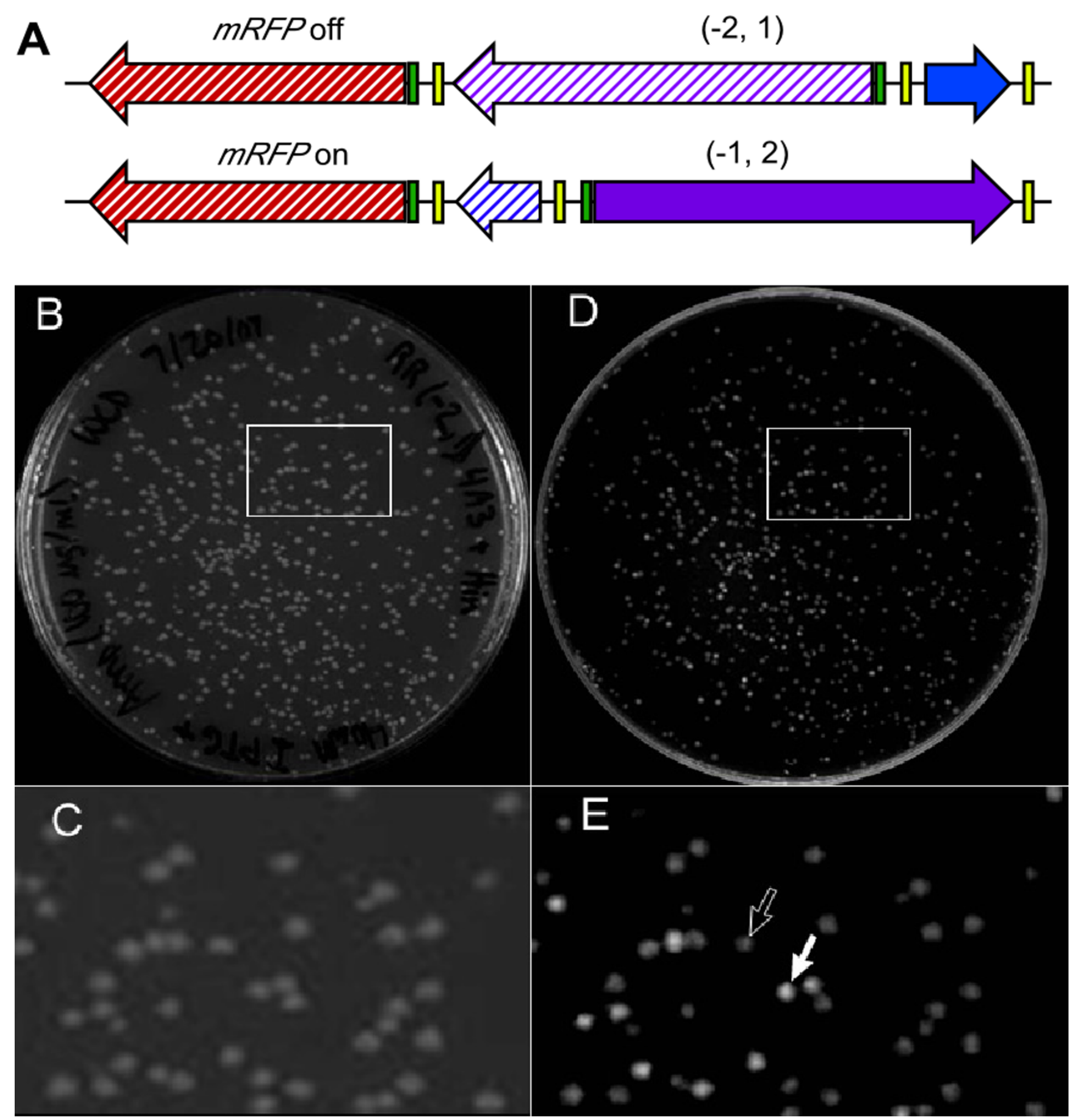

\section{Figure 5}

HinLVA flips adjacent hixC-flanked segments simultaneously. (A) A map of the BPP plasmid including the RBS-mRFP reporter followed by hixC-tetA $(C)_{\text {rev }}$-hixC-pLac-hixC $(-2, I)$ is shown at the top. In this arrangement, pLac cannot drive $m R F P$ expression. Simultaneous inversion of both segments converts $(-2, I)$ into $(-I, 2)$; the pLac promoter is directed towards $m R F P$ so that $m R F P$ expression is turned on. ( $B$, inset $C$ ) White light photograph of colonies 18-hours after cotransformation with BPP plasmid $(-2, I)$ and HinLVA. (D, inset E) mRFP protein production visualized under ultraviolet light (solid white arrow) indicates a simultaneous flip of two adjacent pancakes that placed pLac in the reverse orientation adjacent to $R B S$ - $m R F P_{\text {rev }}$. Some colonies do not glow red (open arrow), indicating a lack of double segment flipping or subsequent conversion into other arrangements that lack mRFP expression (e.g., $(I, 2))$. 
Table I: Two-pancake permutations and their phenotypes

\begin{tabular}{|c|c|c|c|c|c|}
\hline \multirow[t]{2}{*}{ BPP Plasmid construct ${ }^{a}$} & \multirow[t]{2}{*}{ Perm. ${ }^{b}$} & \multicolumn{2}{|c|}{ mRFP expression on amp, IPTGc } & \multicolumn{2}{|c|}{ Growth on tet, amp, IPTGd } \\
\hline & & expected & observed & expected & observed \\
\hline RR-hixC-pLac-hixC-RBS-tetA(C)-hixC & $(1,2)$ & - & - & + & weak \\
\hline$R R$-hixC-tetA $(C)_{\text {rev }}-R B S_{\text {rev }}-h i x C$-pLac rev $_{\text {rev }}-h i x C$ & $(-2,-1)$ & + & - & + & + \\
\hline$R R$-hixC-pLac-hixC-tet $A(C)_{\text {rev }}-R B S_{\text {rev }}-$ hixC & $(I,-2)$ & - & - & - & weak \\
\hline$R R$-hixC-RBS-tetA(C)-hixC-pLac ${ }_{\text {rev }}-h i x C$ & $(2,-1)$ & + & - & - & - \\
\hline RR-hixC-pLac rev $_{\text {rev }}$ hixC-RBS-tetA(C)-hixC & $(-1,2)$ & + & + & - & + \\
\hline$R R$-hixC-tetA $(C)_{\text {rev }}-R B S_{\text {rev }}-h i x C$-pLac-hixC & $(-2,1)$ & - & - & - & + \\
\hline$R R$-hixC-pLac rev $-h i x C$-tet $A(C)_{\text {rev }}-R B S_{\text {rev }}-h i x C$ & $(-1,-2)$ & + & + & - & - \\
\hline RR-hixC-RBS-tetA(C)-hixC-pLac-hixC & $(2,1)$ & - & - & - & + \\
\hline
\end{tabular}

a Two-pancake BPP constructs in plasmid PSB4A3.

$b$ Signed permutation corresponding to each BPP construct.

c Cells carrying a BPP plasmid were selected on ampicillin (amp) plates. IPTG-induced expression of mRFP was visually detected as fluorescent red color (+).

d Serial dilutions of colonies showed normal (+), weak, or no $(-)$ growth on tetracycline (tet) upon IPTG-induced tetA(C) expression.

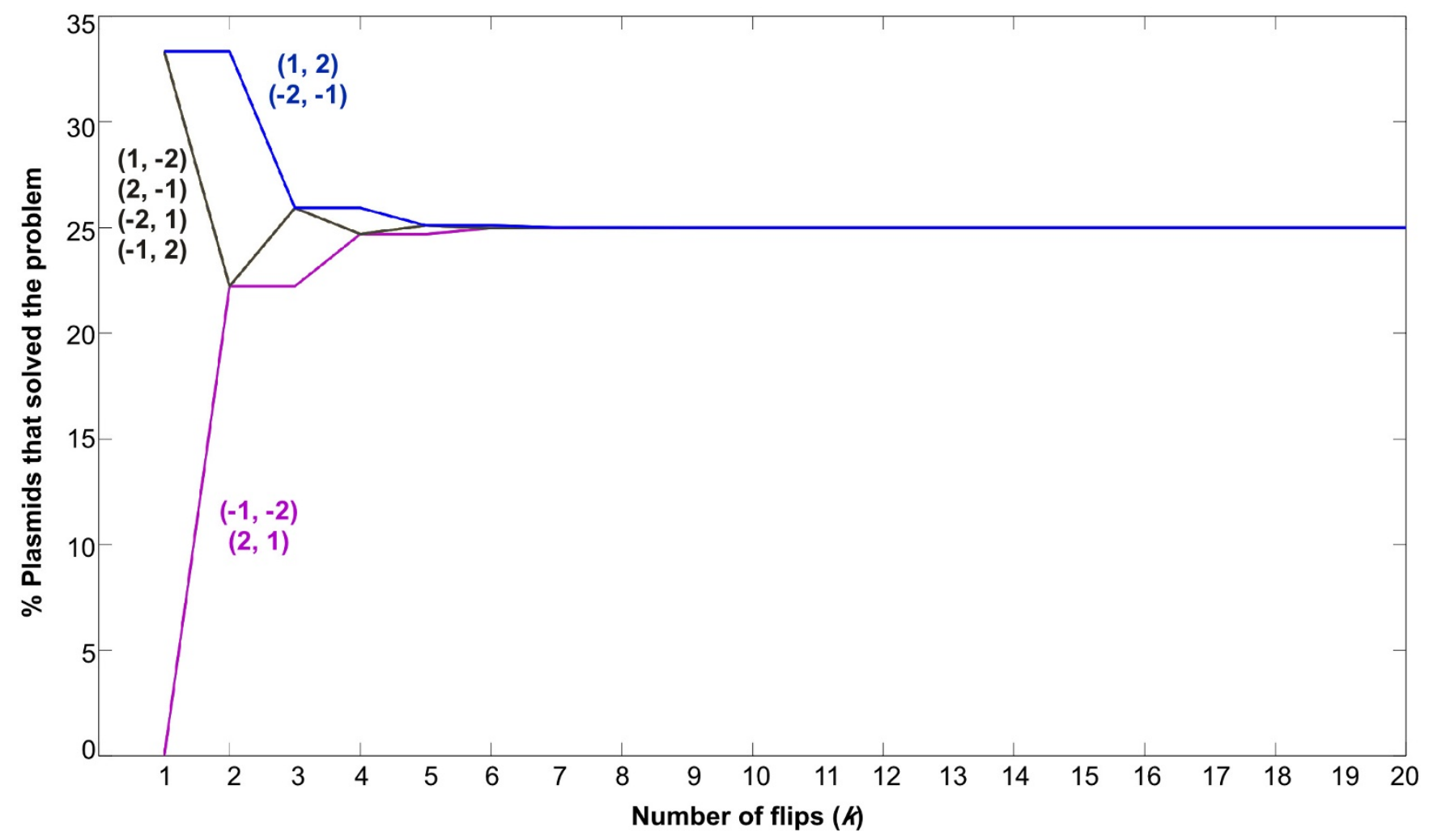

\section{Figure 6}

Mathematical model of random flipping. Flipping was modeled as a Markov Chain in which each of the possible eight starting permutations is a state (shown in parenthesis in the graph). At each step (Number of flips (k)) in a random walk on the graph in Figure Ib, the probability of a plasmid being properly sorted (\% Plasmids that solved the problem) after $k$ flips is calculated as the number of paths of length $k$ from the initial state to the solution state, divided by the total number of paths of length $k$ from the initial state to any state. Starting permutations that show equivalent behavior can be grouped into three families (distinguished by color in the graph). The families are distinguishable for up to $4 \mathrm{flips}$; at 5 flips and beyond they reach a state of equilibrium at $25 \%$ plasmids solved. 
gene and should lead to cell death in the presence of tetracycline. Based on these predicted phenotypic outputs, we designed a mathematical model of random flipping over time (successive flips) to predict how cell survival (the percentage of solved pancake stacks) might change over time. We modeled flipping as a Markov Chain in which each of the possible eight signed permutations is a state. Our model is synonymous with a random walk on the graph in Figure 1b. We assumed that any segment of DNA flanked by two hixC sites (pancake 1, pancake 2, or both) is equally likely to be flipped by HinLVA and that all flips in the population of cells happen synchronously. According to this model, the probability of a plasmid being properly sorted after $k$ flips is determined by the number of paths of length $k$ from the initial state to the solution state, divided by the total number of paths of length $k$ from the initial state to any state. For instance, there are six paths of length 3 from initial state $(2,1)$ to solution state $(1,2)$ (Fig. 1b); because there are 27 possible paths of length 3 that start at $(2,1)$, the probability of being in a solution state after three flips is $6 / 27(22 \%)$. We observed two interesting features of the output from a simulation of random flipping (Fig. 6). First, the conversion of unsolved BPP plasmids towards and away from the solution state reaches equilibrium at $25 \%$ survival after five flips. Second, several starting arrangements show equivalent behavior as they approach equilibrium (i.e., $(1,-2)$ and $(-1,2))$. The simulation output has implications for further design of our system. If our model is correct, only one representative from each class of equivalent starting configurations needs to be tested. Furthermore, if equilibrium (25\% survival) is reached after only five flips, slowing Hin-mediated inversion by omitting the RE may be required to detect significant changes in cell survival over time.

As an initial step towards carrying out flipping in vivo, we manually constructed all eight pancake permutations (excluding the $R E$ ) and transformed them into cells to confirm their phenotypes. We observed several unexpected outcomes. In cells that contain a strong pLac repressor $\left(\right.$ lacl $\left.{ }^{Q}\right)$, BPP plasmids $(1,2)$ and $(-2,-1)$ showed significant tetracycline resistance without activation of pLac by IPTG. We also observed that HinLVA-mediated inversion does not require induction of the pLac promoter on the HinLVA plasmid, indicating general leakiness of pLac promoter activity probably due to more lacIQ binding sites than available repressor protein [18]. The addition of IPTG appears to slow the growth of $(1,2)$ transformants; this might be result of toxic TetA(C) over expression [19]. We expected to detect $m R F P$ expression from all four plasmids that contain reversed $p L a c$. However, reversed $p L a c$ fails to induce $m R F P$ expression when it is positioned after tetA(C) (i.e., RBS-mRFP-hixC-RBS-tetA(C)-hixC-pLac rev ${ }^{-}$
hixC). Increased distance from $m R F P$ or the DNA structure of tetA(C) $[20,21]$ might block transcription of $m R F P$.

We found it surprising that four constructs in which pLac is not in the proper position and/or orientation to drive expression of tetA(C) were able to confer tetracycline resistance; in the presence of IPTG, three of these showed more robust growth than cells carrying $(1,2)$. When the pLac promoter was removed from the construct, cells were still tetracycline resistant (data not shown), thus $p L a c$ is not required for expression in the pBR322-derived cloning vector we had been using (pSB4A3). Read-through transcription by RNA polymerase binding to the antibiotic resistance marker promoter or degenerate promoter sequences within the vector backbone [22] could result in tet $A(C)$ expression in the tetracycline resistant scrambled permutations $(1,-2),(-1,2),(-2,1)$, and $(2,1)$. We constructed an "insulated" vector (pSB1A7) containing forward and reverse double transcription terminator sequences to shield RBS-tet $A(C)$ from read-through transcription. In pSB1A7, there was no expression of $R B S$ tet $A(C)$ (forward or reverse) when $p L a c$ was removed from the construct. Arrangements $(1,2)$ and $(-2,-1)$ produced tetracycline resistance, as expected. Surprisingly, we also observed tetracycline resistance in the insulated vector when $p L a c$ was reversed relative to tetA(C) in arrangements $(-1,2)$ and $(-2,1)$, suggesting reverse promoter activity from $p L a c$. Unlike forward transcription initiated from $p L a c$, backwards transcription did not respond to IPTG as determined by cell growth; IPTG induction of forward transcription from pLac led to overexpression of tetA(C) and subsequent cell death [19]. Due to the backwards promoter activity of $p L a c$, our manually built set of permutations are not distinguishable by phenotype, thus phenotype alone is insufficient to perform computation using $p L a c$ and RBS-tetA(C). The observations described above demonstrate that the construction of synthetic biological devices can reveal unexpected characteristics of well-studied DNA elements (e.g., pLac).

\section{Conclusion}

We have demonstrated that a modified Hin/hix DNA recombination system can be used in vivo to manipulate at least two adjacent hixC-flanked DNA segments; HinLVA and hixC are sufficient for DNA inversion activity. The RE is not required, although it may play some role in preventing aberrant flips that lead to plasmid knotting [23] and subsequent plasmid loss [24]. Thus, the RE might be added to BPP plasmids to increase DNA recombination efficiency. Once phenotypic output is optimized for this system, the kinetics of flipping (i.e., number of flips per unit of time) could be determined by comparing Markov Chain model simulation output to in vivo pancake sorting. Comparing actual cell survival to the survival probabilities predicted by our model should also enable us to deter- 
mine whether flipping is biased for different sized DNA fragments.

The Hin/hix DNA recombination system could be used for other biological engineering applications. We have developed a set of modular genetic elements (hixC, $R E$, and HinLVA) that expands the repertoire of molecular tools for enzyme-mediated DNA manipulation in vivo. As with Cre/loxP from P1 bacteriophage [25] and Flp/FRT from yeast [26], Hin/hix may open avenues for recombinasemediated transgene engineering. For instance, hixCflanked promoters and other regulatory elements could function as flippable genetic toggle switches to regulate gene expression just as Hin mediates the expression of flagellin genes in Salmonella. Manipulation of genetic elements within a transgene at a single insertion site eliminates the problem of genomic position effects associated with independently introducing variants of transgenes at different loci. Furthermore, adjacent genetic elements could be rearranged at a single locus (e.g., switching the positions of a promoter and transcriptional insulator to test how well the insulator blocks transcription). The ability of Hin recombinase to invert large and small DNA fragments and adjacent flippable elements demonstrates the potential flexibility of the Hin/hix system.

The capability of HinLVA to flip adjacent DNA segments indicates that this system could be scaled up to accommodate more complex pancake stacks. As an application in comparative genomics, flippable DNA segment arrays could serve as a model to improve our understanding of syntenic genome rearrangements that have occurred during evolution. Chromosomal regions exist as syntenic modules arranged in different orders and orientations in the genomes of related species. Each syntenic module can be considered a burnt pancake that has a particular order and orientation. Phylogenetic relationships between species can be inferred by using BPP mathematical modeling to compute the minimum number of rearrangements that link two syntenic genomes (see [27] for review). Hinmediated rearrangements of an array containing different sized DNA fragments would help refine the mathematical model by accounting for the impact of differences in sequence composition and lengths of syntenic modules. Using Hin/hix to sort DNA fragment permutations in vivo expands the horizons for the emerging field of applied DNA-based computing.

\section{Methods}

\section{Construction of parts and plasmids}

All genetic elements reported here are standardized parts (prefixed "BBa_" and "pSB") that are flanked by universal "BioBrick" cloning sites [28] and are documented and distributed by the MIT Registry of Standard Biological Parts [29].E. coli strain JM109 was used for cloning and as the chassis for our system. The recombinational enhancer (RE, BBa_J3101) [30], hixC (BBa_J44000) [13], RBS rev (BBa_J44001) and $p B A D_{\text {rev }}\left(\mathrm{BBa} \_\right.$J44002) were assembled from 20 - 60 bp DNA oligomers that were designed using the "Oligo Cuts Optimization Program" [31]. EcoRI (5') and PstI (3') single stranded extensions were manually added to the terminal oligomers. An equimolar mix of single-stranded oligomers in $1 \times$ annealing buffer [100 $\mathrm{mM} \mathrm{NaCl} ; 10 \mathrm{mM}$ Tris- $\mathrm{HCl}, \mathrm{pH}$ 7.4] was incubated at $100^{\circ} \mathrm{C}$ for 5 minutes, then slowly cooled to ambient temperature in a water bath to produce double stranded DNA with EcoRI (5') and PstI (3') single stranded overhangs. The annealed DNA was ligated into linearized (EcoRI and PstI digested) cloning vector $\mathrm{pSB} 1 \mathrm{~A} 2$ using $\mathrm{T} 4$ ligase (Promega). Hin (BBa_J31000), HinLVA (BBa_J31001), and tet $A(C)$ (BBa_J31007), and were cloned by polymerase chain reaction (PCR). Standard BioBrick prefix cloning sites (EcoRI, NotI and XbaI) or suffix cloning sites (SpeI, NotI and PstI) were included in the forward or reverse PCR primer, respectively. PCR amplicons were digested with EcoRI and PstI, electrophoresed in an agarose gel, purified, and ligated into a linearized cloning vector. Hin [GenBank: see Availability and requirements section for URL] was cloned from Salmonella typhimurium Ames strain TA100 genomic DNA using forward primer 5'GCATGAATTCGCGGCCGCTCTAGATGGCTACTATT-

GGGTATATTC and reverse primer 5'-ATGCCTGCAGGCGGCC

GCAACTAGTTAATTCATTCGTTTTTTTATAC. HinLVA was generated by PCR amplification of Hin (BBa_J31000) using forward primer 5'-TCTGGAATTCGCGGCCGCATC TAGAGATG and reverse primer 5'CTGCAGGCGGCCGCTACTAGTATTAAGCTACTAAAGC GTAGTTTTCGTCGTTTGCAGCATTCATTCGTTTTTT-

TATAC containing a ssrA LVA degradation tag (based on $g f p($ down, LVA) [12]).tetA(C) was cloned from vector pSB1AT3 using forward primer 5'-GCATTCTAGATGAAATCTAACAATGCGCTCATC and reverse primer 5'ATGCACTAGTTAGG

TCGAGGTGGCCCGGC; the amplicon was cloned using a $\mathrm{XbaI} /$ SpeI digest. Reversed parts were generated by PCR amplification of MIT Registry parts using a forward primer containing SpeI and a reverse primer containing XbaI: $\operatorname{tet}(\mathrm{C})_{\text {rev }}\left(\mathrm{BBa} \_J 31006\right)$ - forward 5'-ATGCACTAGTATGAAATCTAACAATGCGCTCATC and reverse 5'-GCATTCT AGATTAGGTCGAGGTGGCCCGGC; $p L a c_{r e v}$ (BBa_J31 013) - forward 5'-ATGCACTAGTACAATACGCAAACCGC CTCTC and reverse 5'-GCATTCTAGAGTGTGTGAAATTGTTATCCGC; $m R F P_{r e v}\left(\mathrm{BBa} \_J 31008\right)$ - forward 5'ATGCACTAGTATGGCTTCCTCCGAAGACGT and reverse 5'-GCATTCTAGATTAAGCACCGGTGGAGTGAC. Correct sizes and sequences of the genetic elements described above were confirmed by XbaI/SpeI double digestion and DNA sequencing. The constructs shown in Figure 2, the eight two-pancake BPP plasmids (Table 1), and the Hin- 
LVA expression vector were assembled using the standard BioBrick assembly method [27]. Proper assembly of construction intermediates was confirmed by EcoRI/PstI double digestion. Fully assembled two-pancake BPP constructs $(1,2)$ (BBa_S03684), (-2, -1) (BBa_S03681), $(1,-2)$ (BBa_S03685), (-2, 1) (BBa_S03679), $(-1,2)$ (BBa_S03687), (-2, 1) (BBa_S03680), (2, 1) (BBa_S03677), and $(-1,-2)$ (BBa_S03688) were confirmed by sequencing. BPP constructs were inserted into low copy pSC101 vector pSB4A3 (Fig. 2) and subsequently tested in insulated high copy pMB1 vector pSB1A7. pLac-RBS-HinLVA-TT (BBa_S03536) was inserted into low copy ColE1 vector BBa_J64100 (from Jeffrey J. Tabor) to create the HinLVA plasmid (Fig. 2). The cloning vectors are described in [29].

\section{Hin-mediated DNA recombination}

Growth of transformed colonies on LB agar and in shaking liquid cultures took place overnight at $37^{\circ} \mathrm{C}$. For single DNA segment flipping assays (Fig. 3), constructs containing the HinLVA gene on the same vector as the invertible segment (BBa_J44006 - pLac-RBS-HinLVA-TThixC-pBAD-hixC-RBS-tetA(C)-TT-RE or pLac-RBS-HinLVATT-pBAD-hixC-RBS-tetA(C)-hixC-TT-RE in vector pSB1A2) were transformed into JM109 chemically competent cells. After growth on selective media (LB agar plus $100 \mu \mathrm{g} / \mathrm{mL}$ ampicillin), single colonies were grown in liquid selective media (LB plus $100 \mu \mathrm{g} / \mathrm{mL}$ ampicillin) overnight. For the two-pancake stack flipping assay, 10 ng each of a plasmid containing a two-pancake stack (Table 1 ) and the HinLVA expression plasmid were combined in $5 \mu \mathrm{L}$ sterile distilled $\mathrm{H}_{2} \mathrm{O}$ and co-transformed into Z-competent JM109 chemically competent cells according to the manufacturer's protocol (Zymo Research). Cells were grown at $37^{\circ} \mathrm{C}$ on LB agar selective media $(20 \mu \mathrm{g} / \mathrm{mL}$ ampicillin, $50 \mu \mathrm{g} / \mathrm{mL}$ chlroamphenicol).

\section{Detection of DNA inversions}

Inversions of single hixC-flanked DNA segments were detected by restriction digests (Fig. 3). Plasmid DNA was purified from each culture (Promega Wizard and Zymo Zyppy miniprep systems), digested with NheI, and resolved by agarose (Fig. 3a) or polyacrylamide (Fig. 3b) gel electrophoresis. Inversions of adjacent hixC-flanked segments were detected by multiplex semiquantitative PCR (sqPCR) (Fig. 4). 11 hours following transformation, 46 visible co-transformant colonies were picked from LB agar selective media and subjected to whole cell multiplex sqPCR $\left(95^{\circ} \mathrm{C}, 10 \mathrm{~min}\right.$.; $95^{\circ} \mathrm{C}, 30 \mathrm{sec}$, $60^{\circ} \mathrm{C}, 30 \mathrm{sec}$., and $72^{\circ} \mathrm{C}, 15 \mathrm{sec}$. for 26 cycles). Each colony was suspended in $60 \mu \mathrm{L} 1 \times$ PCR mix (Promega Green master mix plus 0.7 $\mu \mathrm{M}$ primers $p L a c R$ 5'-GAATCGGCCAACGCGCGGGG, $p L a c F$ 5'-GTTTCCCGACTGGAAAGCGG，TetR 5'-GTAGAGGATCCACAGGACGG and TetF 5'-TCGTAGGACAGGTGCCGGCA). $0.1 \mathrm{pmol}$ of an equimolar mix of all 8 two-pancake BPP plasmids or $0.1 \mathrm{pmol}$ of the $(-2,1)$ BPP plasmid alone was used as the template in a control PCR reaction. During PCR, an $11 \mu \mathrm{L}$ aliquot was collected from each reaction after cycles 18, 20, 22, 24 and 26. Samples were electrophoresed on a $1.5 \%$ agarose gel and photographed under ultraviolet light using a BioRad imager. Band intensities were quantified using BioRad Quantity One imaging software. The detectable band threshold was set at 10,000 (after background subtraction).

To detect simultaneous flipping of two DNA segments (described in Fig. 5) single co-transformed colonies were picked from agar plates and grown in selective liquid media $(20 \mu \mathrm{g} / \mathrm{mL}$ ampicillin, $50 \mu \mathrm{g} / \mathrm{mL}$ chlroamphenicol). To eliminate the Hin plasmid after flipping, plasmid DNA was purified from the liquid cultures and transformed into new competent cells. Cells were grown on LB agar containing $20 \mu \mathrm{g} / \mathrm{mL}$ ampicillin to select for transformants that contained a recombined BPP plasmid, and $40 \mu \mathrm{g} / \mathrm{mL}$ IPTG to induce $p L a c$-driven expression of $m R F P$. $m R F P$ expression was detected under ultraviolet light and photographed using a BioRad imager.

\section{Availability and requirements}

Genbank: $\quad$ http://www.ncbi.nlm.nih.gov/sites/ent $\underline{\text { rez } ? \text { \&md }=\text { search \&term }=1254295 \& \mathrm{db}=\text { gene }}$

\section{Competing interests}

The authors declare that they have no competing interests.

\section{Authors' contributions}

LJH, TTE, JLP and AMC conceived the original study. KAH, TTE, and AMC constructed pancake plasmids containing pLac, assayed Hin-mediated two-pancake DNA inversions and drafted the manuscript. TTE and KAH assayed Hinmediated single pancake inversions. LJH, JLP, WLH, JOD, and $\mathrm{MB}$ designed and executed mathematical modeling of the BPP and DNA flipping. KAH, BJO, SR, ADB, SS, TLB, EZ, AMC, TTE, ELJ, and KJM constructed and confirmed parts described in Methods (Construction of Parts and Plasmids). All authors read and approved the final manuscript.

\section{Acknowledgements}

Vector BBa_64100 was generously provided by J. Tabor. KAH, WLH, JOD and EZ were supported by HHMI grant 52005 I 20. SR was supported by a grant from iGEM at the Massachusetts Institute of Technology. ADB, MLB, TLB, ELJ KJM, BJO, JLP, LHH, and TTE were supported by the Missouri Western State University Summer Research Institute. LJH and AMC worked pro bono. KAH, AMC, LJH, and TTE are members of the Genome Consortium for Active Teaching [32]. We thank the reviewers for their help with improving the final manuscript. 


\section{References}

I. Adleman LM: Molecular computation of solutions to combinatorial problems. Science 1994, 266:1021-1024.

2. Benenson Y, Paz-Elizur T, Adar R, Keinan E, Livneh Z, Shapiro E: Programmable and autonomous computing machine made of biomolecules. Nature 200I, 4I4:430-434.

3. Soreni M, Yogev S, Kossoy E, Shoham Y, Keinan E: Parallel biomolecular computation on surfaces with advanced finite automata. J Am Chem Soc 2005, I 27:3935-3943.

4. Kossoy E, Lavid N, Soreni-Harari M, Shoham Y, Keinan E: A programmable biomolecular computing machine with bacterial phenotype output. Chembiochem 2007, 8: I255-I 260.

5. Gates $\mathrm{WH}$, Papadimitriou $\mathrm{CH}$ : Bounds for sorting by prefix reversal. Discrete Math 1979, 27:47-57.

6. Bafna V, Pevzner P: Sorting by reversals: Genome rearrangements in plant organelles and evolutionary history of $X$ chromosome. Molecular Biology and Evolution 1995, I 2:239-246.

7. Hannenhalli S, Pevzner PA: Transforming Cabbage into Turnip: polynomial algorithm for sorting signed permutations by reversals. Journal of the ACM 1995, 46: I-27.

8. Bourque G, Pevzner PA, Tesler G: Reconstructing the genomic architecture of ancestral mammals: lessons from human, mouse, and rat genomes. Genome Res 2004, I4:507-5I6.

9. Zieg J, Silverman M, Hilmen M, Simon M: Recombinational switch for gene expression. Science 1977, 196: I70-172.

10. Zieg J, Simon M: Analysis of the nucleotide sequence of an invertible controlling element. Proc Natl Acad Sci USA 1980, 77:4196-4200.

II. Johnson RC, Bruist MF: Intermediates in Hin-mediated DNA inversion: a role for Fis and the recombinational enhancer in the strand exchange reaction. EMBO J 1989, 8:I58I-1590.

12. Andersen JB, Sternberg C, Poulsen LK, Bjorn SP, Givskov M, Molin S: New unstable variants of green fluorescent protein for studies of transient gene expression in bacteria. Appl Environ Microbiol 1998, 64:2240-2246.

13. Lim HM, Hughes KT, Simon MI: The effects of symmetrical recombination site hix $\mathrm{C}$ on $\mathrm{Hin}$ recombinase function. J Biol Chem 1992, 267: I I I83-I I 190.

14. Moskowitz IP, Heichman KA, Johnson RC: Alignment of recombination sites in Hin-mediated site-specific DNA recombination. Genes Dev 1991, 5: 1635-1645.

15. Johnson RC, Bruist MB, Glaccum MB, Simon MI: In vitro analysis of Hin-mediated site-specific recombination. Cold Spring Harb Symp Quant Biol 1984, 49:751-760.

16. Lim HM, Simon MI: The role of negative supercoiling in Hinmediated site-specific recombination. J Biol Chem 1992, 267: I I 176-I| I82.

17. Merickel SK, Johnson RC: Topological analysis of Hin-catalysed DNA recombination in vivo and in vitro. Mol Microbiol 2004, 5 I: I |43-I I 54.

18. Glascock CB, Weickert MJ: Using chromosomal laclQI to control expression of genes on high-copy-number plasmids in Escherichia coli. Gene 1998, 223:221-231.

19. Eckert B, Beck CF: Overproduction of transposon TnI0encoded tetracycline resistance protein results in cell death and loss of membrane potential. J Bacteriol 1989, | 7 |:3557-3559.

20. Pruss GJ, Drlica K: Topoisomerase I mutants: the gene on pBR322 that encodes resistance to tetracycline affects plasmid DNA supercoiling. Proc Natl Acad Sci USA 1986, 83:8952-8956.

21. Shishido K, Ishii S, Komiyama N: The presence of the region on pBR322 that encodes resistance to tetracycline is responsible for high levels of plasmid DNA knotting in Escherichia coli DNA topoisomerase I deletion mutant. Nucleic Acids Res 1 989, I 7:9749-9759.

22. Sassone-Corsi P, Corden J, Kedinger C, Chambon P: Promotion of specific in vitro transcription by excised "TATA" box sequences inserted in a foreign nucleotide environment. Nucleic Acids Res 198I, 9:394I-3958.

23. Heichman KA, Moskowitz IP, Johnson RC: Configuration of DNA strands and mechanism of strand exchange in the Hin invertasome as revealed by analysis of recombinant knots. Genes Dev 1991, 5:1622-1634.
24. Deibler RW, Mann JK, de Sumners WL, Zechiedrich L: Hin-mediated DNA knotting and recombining promote replicon dysfunction and mutation. BMC Mol Biol 2007, 8:44.

25. Gu H, Marth JD, Orban PC, Mossmann H, Rajewsky K: Deletion of a DNA polymerase beta gene segment in $T$ cells using cell type-specific gene targeting. Science 1994, 265:103-106.

26. Broach JR, Hicks JB: Replication and recombination functions associated with the yeast plasmid, 2 mu circle. Cell 1980, 21:501-508.

27. Hayes B: Sorting Out the Genome. American Scientist 2007, 95:386-391.

28. Knight T: Idempotent Vector Design for Standard Assembly of Biobricks. 2007.

29. MIT Registry of Standard Biological Parts [http://partsregis try.org]

30. Perkins-Balding D, Dias DP, Glasgow AC: Location, degree, and direction of DNA bending associated with the Hin recombinational enhancer sequence and Fis-enhancer complex. J Bacteriol 1997, I 79:4747-4753.

31. Oligo Cuts Optimization Program [http://gcat.davidson.edu/ IGEM06/oligo.html]

32. Genome Consortium for Active Teaching [http:// www.bio.davidson.edu/GCAT]
Publish with Bio Med Central and every scientist can read your work free of charge

"BioMed Central will be the most significant development for disseminating the results of biomedical research in our lifetime. "

Sir Paul Nurse, Cancer Research UK

Your research papers will be:

- available free of charge to the entire biomedical community

- peer reviewed and published immediately upon acceptance

- cited in PubMed and archived on PubMed Central

- yours - you keep the copyright

Submit your manuscript here:

http://www.biomedcentral.com/info/publishing_adv.asp
BioMedcentral 